University of Nebraska - Lincoln

DigitalCommons@University of Nebraska - Lincoln

$5-15-2003$

\title{
Exchange through nonmagnetic insulating matrix
}

\author{
Ralph Skomski \\ University of Nebraska-Lincoln, rskomski2@unl.edu \\ Arti Kashyap \\ University of Nebraska-Lincoln, akashyap@Inmiit.ac.in \\ Y. Quiang \\ University of Nebraska - Lincoln \\ David J. Sellmyer \\ University of Nebraska-Lincoln, dsellmyer@unl.edu
}

Follow this and additional works at: https://digitalcommons.unl.edu/physicssellmyer

Part of the Physics Commons

Skomski, Ralph; Kashyap, Arti; Quiang, Y.; and Sellmyer, David J., "Exchange through nonmagnetic insulating matrix" (2003). David Sellmyer Publications. 34.

https://digitalcommons.unl.edu/physicssellmyer/34

This Article is brought to you for free and open access by the Research Papers in Physics and Astronomy at DigitalCommons@University of Nebraska - Lincoln. It has been accepted for inclusion in David Sellmyer Publications by an authorized administrator of DigitalCommons@University of Nebraska - Lincoln. 


\title{
Exchange through nonmagnetic insulating matrix
}

\author{
R. Skomski, ${ }^{\text {a) }}$ A. Kashyap, Y. Qiang, and D. J. Sellmyer \\ Department of Physics and Astronomy and Center of Material Research and Analysis, University of \\ Nebraska, Lincoln, Nebraska 68588
}

(Presented on 12 November 2002)

\begin{abstract}
Exchange interactions between hard-magnetic particles in a nonmagnetic matrix are investigated by model calculations. A Landau-Ginzburg approach is developed to describe the net exchange interactions between spheres of arbitrary diameters. Introducing cylindrical coordinates and integrating over the surfaces of the adjacent spheres yields an exchange coupling which decreases with a decay length depending on interatomic exchange, intra-atomic exchange, and temperature. Typically, the decay length does not exceed a few interatomic distances. The decay is exponential but also contains a prefactor depending on the surface curvature of the grains. It increases with decreasing curvature, but this dependence is only a small correction to the leading exponential term.

(C) 2003 American Institute of Physics. [DOI: 10.1063/1.1541633]
\end{abstract}

\section{INTRODUCTION}

Nanostructuring has long been considered and used as a tool to improve the performance of permanent magnets. ${ }^{1-3}$ One approach is to improve the coercivity by making the reversal mechanism more Stoner-Wohlfarthlike, so that the coercivity approaches the anisotropy field $H_{a}$ $=2 K_{1} / \mu_{o} M_{s}$. This can be achieved, for example, by embedding the particles in paramagnetic matrix. However, to realize Stoner-Wohlfarth behavior it is necessary to suppress interparticle interactions, which lead to cooperative magnetization reversal and often to a pronounced coercivity reduction. These interactions can be avoided by embedding the particles in a paramagnetic matrix, but the high packing fractions necessary to realize useful energy products means that particles should nearly touch each other. This limits the usefulness of particle separation to reduce interactions. To gauge this effect, it is necessary to determine the net exchange interactions between particles in a paramagnetic matrix.

There are two main groups of materials of interest. First, various types of $\mathrm{Sm}-\mathrm{Co}$-based magnets, such as $\mathrm{Sm}-\mathrm{Co}-$ $\mathrm{Cu}-\mathrm{Zr}$ (Ref. 4) and $\mathrm{Sm}-\mathrm{Co}-\mathrm{Cu}-\mathrm{Ti}$ (Ref. 5) exhibit a coercivity maximum at elevated temperatures. The main coercivity mechanism in these types of magnets is of the pinning type. ${ }^{6,7}$ Zhou et al. ${ }^{5}$ found that the main origin of the coercivity maximum is the temperature dependence of the anisotropy difference $\Delta K=\left|K_{1: 5}-K_{2: 17}\right|$ between the main 2:17 and grain-boundary 1:5 phases. An alternative explanation is a thermal decoupling of the grains above the Curie temperature of the grain-boundary phase. ${ }^{8,9}$ In fact, these two mechanisms are complementary rather than exclusive and have a common origin, namely that intersublattice exchange is un-

\footnotetext{
${ }^{a)}$ Electronic mail: rvdskomski@msn.com
}

able to suppress rare-earth intramultiplet excitations at temperatures comparable to $T_{C} \cdot 3,10$

Second, embedding magnetic particles or clusters in a nonmagnetic matrix tends to enhance the coercivity and may be used to fabricate artificially structured permanent magnets. As briefly discussed by Sellmyer et al.,${ }^{11}$ this mechanism is closely related to the aforementioned grain decoupling caused by a paramagnetic grain-boundary phase. A similar though undesired coercivity enhancement is observed in soft-magnetic two-phase alloys, where the coercivity reaches a maximum at the Curie temperature of the amorphous grain-boundary phase. ${ }^{12}$

For magnetic nanoparticles embedded in a Pauliparamagnetic matrix, the Ruderman-Kittel-Kasuya-Yosida (RKKY) interaction has been investigated. ${ }^{13}$ There, the net exchange interaction is obtained by adding of, or integrating over atomic RKKY interactions, but this approach is not applicable at finite temperatures and to particles in an insulating matrix. Furthermore, for high volume fractions of the ferromagnetic phase, the corresponding exchange expression becomes very cumbersome, and it is necessary to use approximations. The same is true for ferromagnets above $T_{C}$ (Curie-Weiss paramagnets) and nonmagnetic media. In this article, a Landau-Ginzburg approach is developed to describe the net exchange interactions between neighboring spheres of arbitrary diameters.

\section{CALCULATION AND RESULTS}

To investigate the exchange interaction between grains in an insulating matrix, we consider the geometry shown in Fig. 1, where $R$ indicates the particle radius and $d_{\min }$ is the interparticle distance. The model energy as a function of the magnetization, $m$, is 


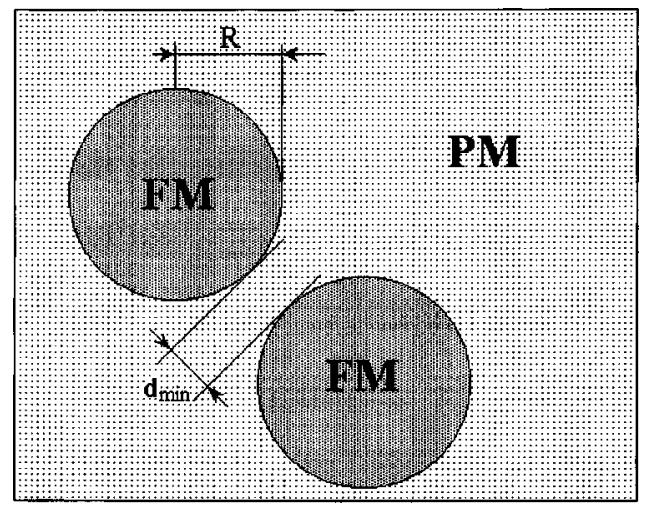

FIG. 1. Considered geometry. Ferromagnetic particles are embedded in a paramagnetic matrix.

$$
E=\frac{1}{2} \int\left[\frac{J}{a}(\nabla m)^{2}+\frac{I_{\mathrm{eff}}(T)}{a^{3}} m^{2}\right] d V
$$

where $J$ is the interatomic exchange, $I_{\text {eff }}(T)$ is an intra-atomic exchange parameter, and $a$ is the interatomic distance. Equation (1) corresponds to a magnetic decay length $1 / \kappa$, where $\kappa^{2}=I_{\text {eff }}(T) / J a^{2}$. Essentially, Eq. (1) is of the LandauGinzburg type, ${ }^{14}$ and $\kappa$ is of the order of $10 / \mathrm{nm}$ in typical insulators such as $\mathrm{Al}_{2} \mathrm{O}_{3} \cdot{ }^{15}$

For two planes separated by a nonmagnetic medium of thickness $d_{o}$, Eq. (1) leads to the differential equation

$$
\frac{d^{2} m}{d x^{2}}+\kappa^{2}=0 .
$$

For the boundary conditions $m\left(-d_{o} / 2\right)= \pm m_{o}$ and $m$ $\left(+d_{o} / 2\right)=+m_{o}$, the respective solutions of Eq. (2) are

$$
m_{+}(x)=m_{o} \frac{\cosh (\kappa x)}{\cosh \left(\kappa d_{o} / 2\right)},
$$

and

$$
m_{-}(x)=m_{o} \frac{\sinh (\kappa x)}{\sinh \left(\kappa d_{o} / 2\right)} .
$$

The dashed (ferromagnetic) and solid (antiferromagnetic) lines in Fig. 2 show the magnetization profiles given by Eqs.

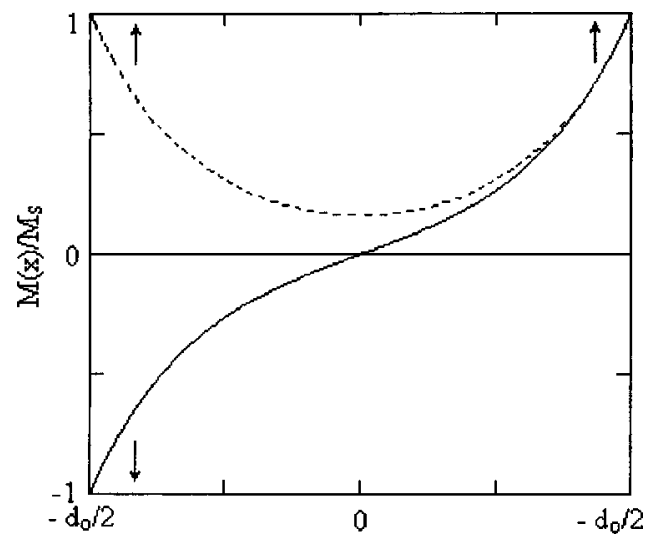

FIG. 2. Spin polarization in a nonmagnetic insulating matrix for ferromagnetic coupling (dashed line) and antiferromagnetic coupling (solid line).

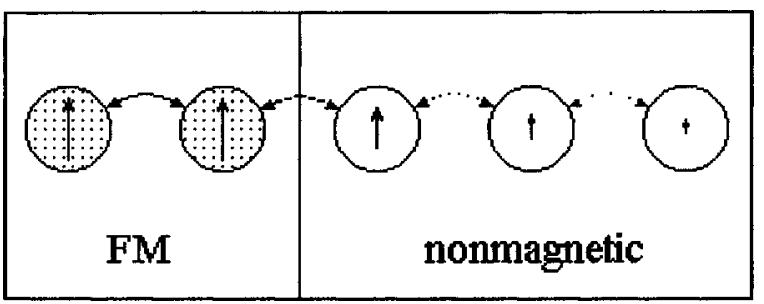

FIG. 3. Distance dependence of the interatomic exchange (schematic). The dotting of the arrows shows the strength of the exchange.

3(a) and 3(b), respectively. Putting the two profiles into Eq. (1) yields a small energy difference, which is equal to the interparticle exchange per surface area.

The net interparticle exchange energy $J_{\text {ip }}$ is obtained from Eqs. (1) and (3). Exploiting the rotational symmetry of the problem, it is convenient to write $J_{\text {ip }}=2 \pi \int \eta(r) r d r$, where the average energy density, $\eta=\eta_{-}-\eta_{+}$is obtained by $x$ integration from $-d_{o} / 2$ to $d_{o} / 2$. After short calculation, we obtain

$$
\begin{aligned}
\eta= & \frac{I_{\mathrm{eff}}(T) m_{o}^{2}}{2 a^{2} \kappa} \sinh \left(\kappa d_{o}\right)\left(\frac{1}{\sinh ^{2}\left(\kappa d_{o} / 2\right)}\right. \\
& \left.-\frac{1}{\cosh ^{2}\left(\kappa d_{o} / 2\right)}\right)
\end{aligned}
$$

and

$$
\eta=\frac{2 I_{\mathrm{eff}}(T) m_{o}^{2}}{a^{2} \kappa} \frac{1}{\sinh \left(\kappa d_{o}\right)} .
$$

Since the inverse decay length is quite large, $\kappa \approx 10 / \mathrm{nm}$ for typical insulators, the hyperbolic sine can be approximated by an exponential function, so that

$$
\eta=\frac{4 I_{\mathrm{eff}}(T) m_{o}^{2}}{a^{2} \kappa} \exp \left(-\kappa d_{o}\right) .
$$

Next, we take into account that the particle radius $R$ is much larger than $1 / \kappa$, so that the distance $d_{o}=d_{\min }+2\left[R-\left(R^{2}\right.\right.$ $\left.\left.-r^{2}\right)^{1 / 2}\right]$ is in good approximation $d_{o}=d_{\min }+r^{2} / R$. The integration over $r$ can then be performed analytically, and we obtain

$$
J_{\text {ip }}=\frac{4 \pi I_{\text {eff }}(T) R m_{o}^{2}}{a^{3} \kappa^{2}} \exp \left(-\kappa d_{\text {min }}\right),
$$

or

$$
J_{\text {ip }}=4 \pi m_{o}^{2} J \frac{R}{a} \exp \left(-\kappa d_{\text {min }}\right) .
$$

When the radii of the two grains are different $\left(R_{1}\right.$ and $\left.R_{2}\right)$, then $R$ must be replaced by $R_{\text {eff }}=2 R_{1} R_{2} /\left(R_{1}+R_{2}\right)$. Since the exchange energy $J_{\text {ip }}$ competes against the anisotropy energy, $K_{1} V$, it is instructive to consider the exchange-energy density $J_{\mathrm{vol}}=J_{\text {ip }} / V$ rather the $J_{\text {ip }}$ itself

$$
J_{\mathrm{vol}}=\frac{3 m_{o}^{2} J}{a R^{2}} \exp \left(-\kappa d_{\mathrm{min}}\right) .
$$


Equation (6) describes the net exchange between two particles. Since the integration leading from Eq. (5) to Eq. (6) assumes nearly plane adjacent surface areas, this result is limited to small spacings $d_{\min }$.

\section{DISCUSSION AND CONCLUSIONS}

The strong decrease of the exchange with increasing interparticle spacing, as epitomized by the decay length $1 / \kappa$ $\approx 1 \AA$, reflects the weakness of the interatomic exchange as compared to the intra-atomic exchange. Figure 3 illustrates that the interatomic exchange creates a small moment in neighboring nonmagnetic atoms, and the exchange between these moments leads to the net exchange. The number of adjacent pairs of nanoparticle surface atoms is quite large and leads to the factor $R$ in Eq. (6b), but this factor gives only a minor correction to the leading exponential term and significant exchange is limited to a few interatomic distances. In permanent magnets, this amounts to a nearly complete decoupling if the particles do not touch, whereas in soft magnets, an effective coupling is limited to a very few $\mathrm{nm}$. For example, assuming $R=10 \mathrm{~nm}, J / k_{B}=1000 \mathrm{~K}, \quad a$ $=0.25 \mathrm{~nm}, m_{o}=1$, and $\kappa=10 / \mathrm{nm}$ yields, with Eq. (6c), an interaction energy density of $1 \mathrm{~kJ} / \mathrm{m}^{3}$ at $d_{\min } \approx 0.6 \mathrm{~nm}$. This corresponds to the anisotropy energy of a very soft material.

Equation (6) means that interparticle exchange through a nonmagnetic matrix is important for distances of at most a very few interatomic distances. There are, however, various mechanisms capable of enhancing this range. One example is when the matrix is close to the onset of ferromagnetism (paramagnetism just above $T_{C}$ ). Due to the vicinity of the ferromagnetic instability, the intra-atomic exchange parameter is small and the decay length is large. (The definition used in this work means that the sign of the exchange parameter is negative for ferromagnets.) Similar effects may be caused by disorder, such as magnetic impurities in the matrix or the vicinity of a percolation transition, ${ }^{16}$ and by the presence of specific resonant states.

In conclusion, we have investigated exchange interactions for nanocrystalline materials consisting of hard mag- netic particles embedded in insulating matrix. The net exchange energy has been obtained by introducing cylindrical coordinates and integrating over the surfaces of the adjacent spheres. In fair approximation, the exchange coupling decreases exponentially with decay length, $d_{\min }$. Unless the matrix is close to ferromagnetism, the decay length is at the most a few interatomic distances, but the large number of adjacent surface atoms yields an enhancement of the exchange which is linear with the particle radius.

\section{ACKNOWLEDGMENT}

This work was supported by the AFOSR, ARO, ONR, and CMRA.

${ }^{1}$ R. Coehoorn, D. B. de Mooij, J. P. W. B. Duchateau, and K. H. J. Buschow, J. Phys. (France) 49 C-8, 669 (1988).

${ }^{2}$ K.-H. Müller, J. Schneider, A. Handstein, D. Eckert, P. Nothnagel, and H. R. Kirchmayr, Mater. Sci. Eng., A 133, 151 (1991).

${ }^{3}$ R. Skomski and J. M. D. Coey, Permanent Magnetism (Institute of Physics, Bristol, 1999).

${ }^{4}$ J. F. Liu, T. Chui, D. Dimitar, and G. C. Hadjipanayis, Appl. Phys. Lett. 73, 3007 (1998).

${ }^{5}$ J. Zhou, R. Skomski, C. Chen, G. C. Hadjipanayis, and D. J. Sellmyer, Appl. Phys. Lett. 77, 1514 (2000).

${ }^{6}$ K. Kumar, J. Appl. Phys. 63, R13 (1988).

${ }^{7}$ D. Goll, I. Kleinschroth, W. Sigle, and H. Kronmüller, Appl. Phys. Lett. 76, 1054 (2000)

${ }^{8}$ D. J. Sellmyer, C. P. Luo, Y. Qiang, and J. P. Liu, in Nanomaterials and Magnetic Thin Films, Handbook of Thin Film Materials Vol. 5, edited by H. S. Nalwa (Academic, San Diego, 2002), p. 337.

${ }^{9}$ A. M. Gabay, W. Tang, Y. Zhang, and G. C. Hadjipanayis, Appl. Phys. Lett. 78, 1595 (2001).

${ }^{10}$ R. Skomski, J. Appl. Phys. 83, 6724 (1998).

${ }^{11}$ D. J. Sellmyer, J. Zhou, H. Tang, and R. Skomski, Mater. Res. Soc. Symp. Proc. 674, U5.8.1 (2001).

${ }^{12}$ G. Herzer, IEEE Trans. Magn. 25, 3327 (1989).

${ }^{13}$ R. Skomski, Europhys. Lett. 48, 455 (1999).

${ }^{14}$ P. A. Dowben, W. Hürsch, and M. Landolt, J. Magn. Magn. Mater. 125, 120 (1993).

${ }^{15}$ I. I. Oleinik, E. Y. Tsymbal, and D. G. Pettifor, Phys. Rev. B 62, 3952 (2000).

${ }^{16}$ M. Ohnuma, K. Hono, H. Onodera, S. Ohnuma, H. Fujimori, and J. S. Pedersen, J. Appl. Phys. 87, 817 (2000). 